\title{
250 Jahre Badische Landesbibliothek
}

Um 1500 wurde in Pforzheim die markgräfliche Hofbibliothek gegründet, aus der später die Badische Landesbibliothek (BLB) hervorging. Damit ist die BLB wesentlich älter als die Württembergische Landesbibliothek (WLB) in Stuttgart, die erst im 18. Jahrhundert gegründet wurde. Ein genaues Gründungsdatum für die BLB ist jedoch leider nicht bekannt. Für die WLB kennt man es dagegen ganz genau: An seinem 37. Geburtstag, am 11. Februar 1765 , gründete der württembergische Herzog Karl Eugen (1728-1793) "von den wiederholten Glück- und Seegens Wünschen der Unterthanen an Höchstdero Geburtsfeste empfindlich gerührt" die Öffentliche Bibliothek. Die handschriftlich verfasste und gesiegelte Stiftungsurkunde ist in den Beständen der Bibliothek bis heute vorhanden. ${ }^{1}$

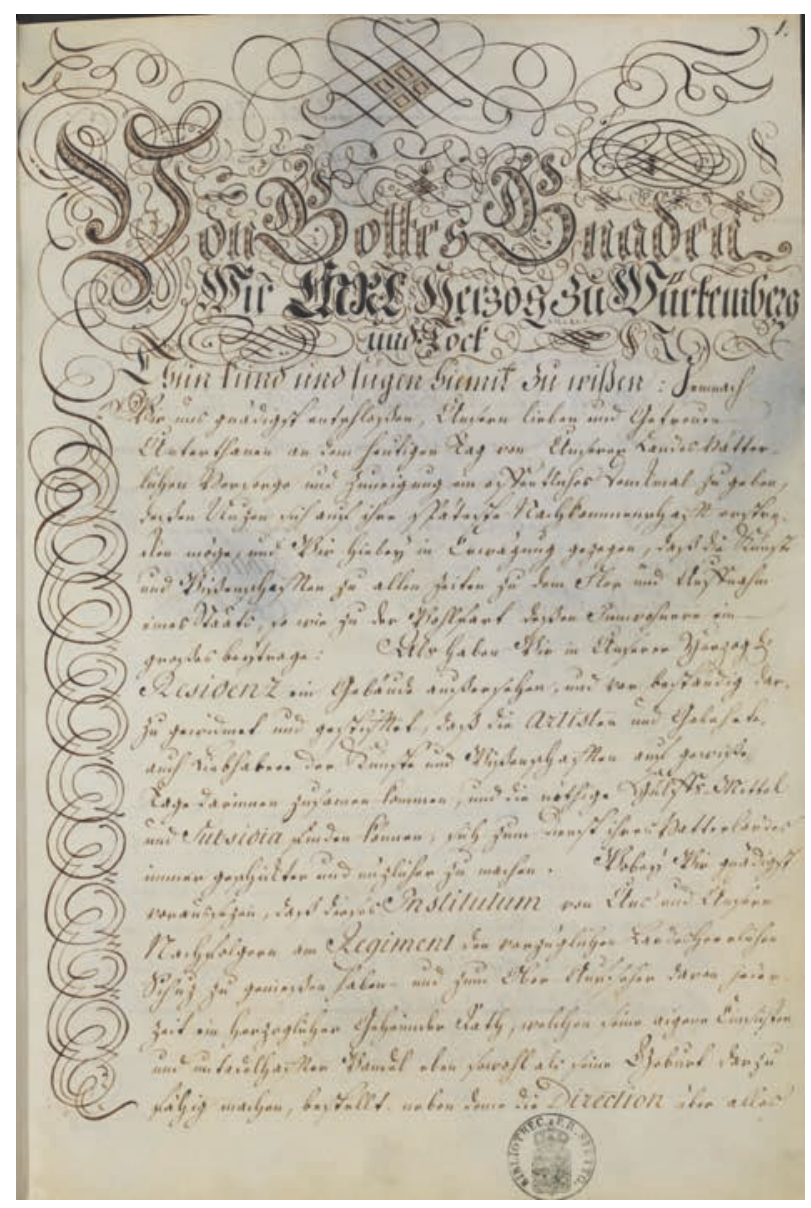

Abb. 1: Stiftungsurkunde der WLB, 11.2.1765
Die Bibliothek der badischen Markgrafen wurde hingegen nach diversen Teilungen und Verlagerungen an unterschiedliche Orte 1771 in Karlsruhe vereinigt. Einen dringenden Handlungsbedarf gab es jedoch bei der Benutzung der Bibliothek. Die Benutzung desjenigen Teils der Bibliothek, der sich schon früher in Karlsruhe befand, war offenbar in Unordnung geraten, denn in einem Geheimen Ratsprotokoll vom 23. Juli 1770 hieß es, mehrmals sei es passiert, "daß die von hiesiger fürstlichen Bibliothek entlehnten [= entliehenen] Bücher theils gar nicht wieder zurückgegeben - theils ungebührlich lang behalten - theils auch in verdorbenem Stand heimgestellt worden seyen".

Markgraf Karl Friedrich (1728-1811) beauftragte nun den Hofrat Friedrich Valentin Molter (17221808) und den Kanzleibeamten Johann Christian Griesbach (1736-1804), eine Denkschrift auszuarbeiten, um die Benutzungssituation der Bibliothek zu verbessern. In dieser Denkschrift (De Germania

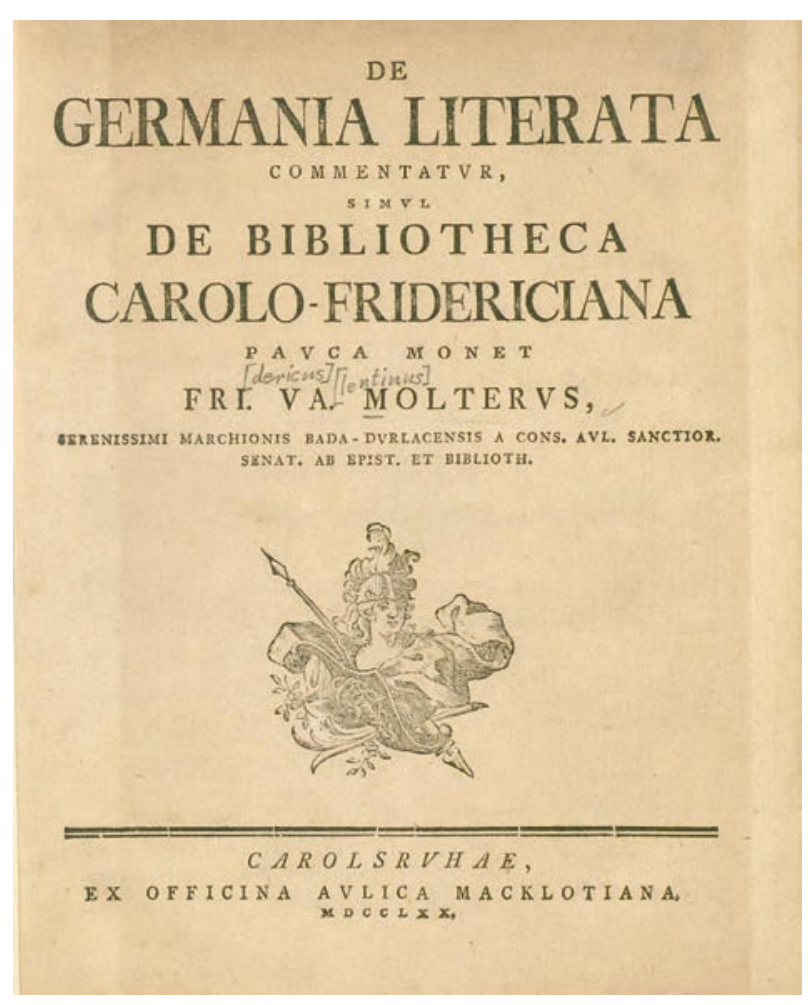

Abb. 2: Molter, Friedrich Valentin: De Germania Literata commentatur simul de bibliotheca Carolo-Fridericiana pauca monet, Karlsruhe: Macklot 1770
1) Urkunde der Stiftung der herzoglichen öffentlichen Bibliothek durch Herzog Carl Eugen (Cod.hist.fol.209) (http://digital.wlb-stuttgart.de/ purl/bsz35103255X).

2) Generallandesarchiv Karlsruhe (GLA) 47, Nr. 1976. 
Literata Commentatvr, Simvl De Bibliotheca Carolo-Fridericiana Pavca Monet ${ }^{3}$ ) wurde vorgeschlagen, den freien Zugriff der Benutzer auf die Bände zu erschweren, die Entleihungen sollten in einem Verzeichnis festgehalten und Handschriften sowie seltene Bücher gar nicht ausgeliehen werden. Um die Bücher als Bände der Bibliothek erkennen zu können, sollten sie Einbände mit dem Wappen des Markgrafen bekommen.

Diese Ratschläge flossen dann in die erste bekannte Benutzungsordnung der Bibliothek ein, die am 31. Dezember 1770 von Markgraf Karl Friedrich unterzeichnet wurde und 1771 in Kraft trat. ${ }^{4}$ Diese Bibliotheksordnung blieb mehrere Jahre in Kraft. Noch 1786 war sie an den Innenseiten der Türflügel des Büchersaales ausgehängt.
In dieser ersten Bibliotheksordnung von 1770/71 wurde zum ersten Mal die öffentliche Zugänglichkeit der Bibliothek festgeschrieben, auch wenn der Begriff der Öffentlichkeit nicht so weit gefasst wurde wie heutzutage. Das zeigen zum Beispiel die Einschränkungen der Benutzung, wie sie in den „Statuten für die Großherzogliche Hofbibliothek in Karlsruhe" von 1843 noch festgeschrieben waren: Hier wurde festgelegt, die Bibliothek sei zunächst „ein Hofinstitut und bleibt zunächst zum Gebrauch der Großherzoglichen Familie bestimmt; jedoch wird ihre öffentliche Benutzung [...] zu amtlichen Berufsarbeiten und zu erwiesenen wissenschaftlichen Zwecken, nicht aber zur Befriedigung bloßer Neugierde oder zum Zeitvertreib gestattet" . ${ }^{5}$ Trotzdem lässt sich sagen: Die heutige Badische Landesbibliothek kann in diesem Jahr 2021 ein wichtiges Jubiläum feiern. Sie ist seit 250

Jahren eine öffentlich zugängliche Bibliothek.

I. Singulis Mercurii \& Saturni diebus Hora matutina X. ad XII. \& poft meridiem Hora III. ad V. vfque Bibliotheca pateto.

II. Libros ex forulis depromere, euoluere, perluftrare iisque carm decenter vti fas efto, quo facto in eumdem illi, quem tenebant quiue Cat.logo adnotatus eft, locum reconduntor.

III. Libri commodati folerter in Diarium librorum commodatorum referuntor, femel rubrica tenus, deinde fub nomine Commodatarii die, quo dabantur, adiecto.

IV. Syngrapham at! Bibliothecam mittito quicumque libros inde petierit domum fibi adferendos.

V. Codices manuferipti aut impreffi rariores ad aedes nifi ponderofis ex cuufis ne commodantor.

VI. Libros fi quis ex Bibliotheca acceperit, is quidem caueto, ne quid illi detrimenti capiant, neue omnimo perdantur; alioquin nouos ipfe propriis fumptibus reddito aut damna-praeftato.

VII. Elapfo quoque menfe libri commodati ad Bibliothecam reportantor, aut fi quis vltra id tempus opus iisdem habeat, nouam fyngrapham dato atque in Diario recens dies notator.
Öffentlich zugänglich waren zuvor noch nicht viele Bibliotheken. Zu diesen wenigen Einrichtungen zählten zum Beispiel die Bibliothek des Kurfürsten Karl Theodor in Mannheim (seit 1763) und auch die Herzogliche Öffentliche Bibliothek in
Abb. 3 und 4: Bibliotheksordnung der BLB von 1770 (rechts) und der WLB von 1765/1776 (links)

3) https://digital.blb-karlsruhe.de/id/5446497.

4) Abgedruckt in: Badenscher gemeinnütziger Hof- und Staatskalender für das Jahr 1786 (Abtl. 2), S. 129-139 (https://digital.blb-karlsruhe.de/ blbihd/periodical/pageview/2169876).

5) Zitiert nach: Syré, Ludger: Die Geschichte der Bibliothek - eine Chronik in Daten und Bildern, in: Römer, Gerhard (Hrsg.): Buch. Leser. Bibliothek. Festschrift der Badischen Landesbibliothek zum Neubau, Karlsruhe: Badische Landesbibliothek 1992, S. 13-31, hier S. 19.

6) Urkunde der Stiftung der herzoglichen öffentlichen Bibliothek durch Herzog Carl Eugen (Cod.hist.fol.209) (http://digital.wlb-stuttgart.de/ purl/bsz35103255X).

7) Ordnungen der öffentlichen Bibliotheck, Stuttgart [zwischen 1765 und 1776] (Signatur: HBFC 6093) (http://digital.wlb-stuttgart.de/purl/ bsz417620500).
Stuttgart, die heutige WLB (seit 1765). Schon Karl Eugen hatte in seiner Stiftungsurkunde festgelegt, die Bibliothek solle "jedermänniglich ohne Unterschied des Rangs oder Standes, mit alleiniger Ausnahm der Livrée-Bedienten, offen seyn" ${ }^{6}$

\section{Auch für Stuttgart ist eine Bibliotheksordnung} bekannt, jedoch ohne genaues Datum. Sie muss zwischen 1765 und 1776 verfasst worden sein. ${ }^{7}$ 
Beide Bibliotheken waren von ihren Öffnungszeiten her viel beschränkter zugänglich als heutige Bibliotheken. Die Karlsruher Bibliothek sollte mittwochs und samstags von 10 bis $12 \mathrm{Uhr}$ und von 15 bis 17 Uhr geöffnet sein. Die Öffnungszeiten der Stuttgarter Bibliothek waren Montag, Mittwoch und Freitag jeweils von 9 bis 12 Uhr und von 15 bis 18 Uhr. Karlsruhe bot somit insgesamt nur acht Öffnungsstunden in der Woche an, Stuttgart immerhin 18 Stunden.

\section{Dafür waren die Vorschriften zur Benutzung in} Karlsruhe wesentlich liberaler. Hier hatten die Benutzer direkten Zugang zu den Büchern, während sie in Stuttgart die Bücher über die Bibliothekare bestellen und möglichst im Lesesaal benutzen mussten. Während man in Karlsruhe für die Ausleihe eines Buches nur einen Leihschein (syngrapha) ausfüllen musste (ein Exemplar eines Leihscheines von 1688 hatte sich bis 1945 erhalten!), war in Stuttgart für die Ausleihe eines Buches nach Hause sogar die Genehmigung des Herzogs (!) erforderlich. In beiden Fällen durften die Bücher

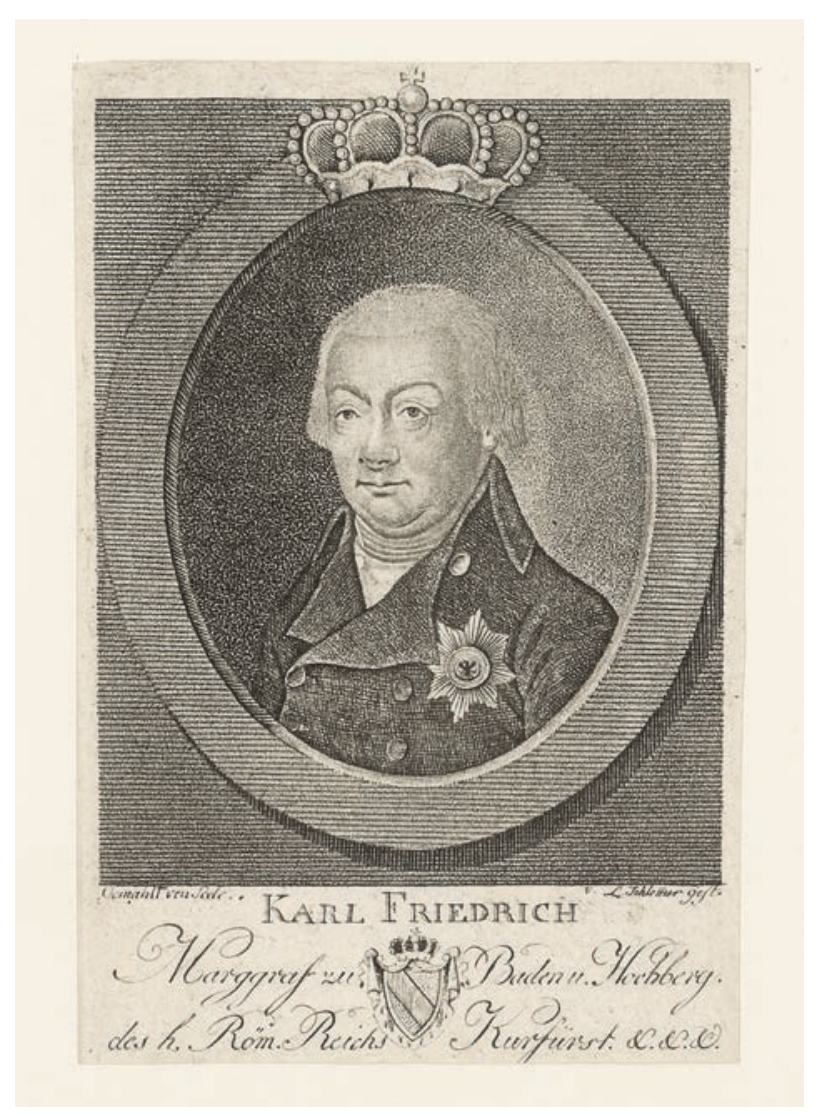

Abb. 5: Markgraf Karl Friedrich von Baden (WLB Stuttgart, Karten und Graphik: Rege.202-5a) einen Monat ausgeliehen werden. In Karlsruhe war auch die Verlängerung der Leihfrist möglich, dazu brauchte es jedoch eines neuen Leihscheins und die neue Leihfrist musste im Ausleihbuch vermerkt werden. Handschriften und, impressi rariores', also seltene Drucke, durften in Karlsruhe nur aus gewichtigen Gründen (ponderosis causis) ausgeliehen werden.

Wie seinen Staat hatte Markgraf Karl Friedrich, dessen Motto "moderate et prudenter" (mäßig und weise) lautete, auch die Benutzung seiner Bibliothek nach liberalen Grundsätzen geordnet. Der württembergische (!) Schriftsteller Christian Friedrich Daniel Schubart (1739-1791), der in seinen Schriften die absolutistische Herrschaft und deren Dekadenz im damaligen Herzogtum Württemberg öffentlich anprangerte, schrieb im Oktober 1774 in seiner Zeitung „Teutsche Chronik": „Baadendurlach gehört seit der weisen Regierung seines itzigen [= jetzigen] Fürsten unter die glücklichsten und besteingerichtetsten Staaten der Welt, auf den andre Provinzen mit nachahmender Eifersucht blicken. [...] Welche Anstalten zur Glückseligkeit des Volks! Welche Ermunterungen zur Gewerbsamkeit, zum Kunstfleiße!"8

Hans-Christian Pust 